\title{
Joint Data-Channel Estimation using the Particle Filtering on Multipath Fading Channels
}

\author{
Tanya Bertozzi ${ }^{* \dagger}$, Didier Le Ruyet ${ }^{\dagger}$, Gilles Rigal * and Han Vu-Thien ${ }^{\dagger}$ \\ * DIGINEXT, 45 Impasse de la Draille, 13857 Aix en Provence Cedex 3, France, \\ Telephone: 00334429082 82, Fax: 00334429082 80, Email: bertozzi@ diginext.fr \\ $\dagger$ Signals and Systems Laboratory, CNAM, 292 rue Saint Martin, 75141 Paris Cedex 3, France \\ Telephone: 00331402727 98, Fax: 0033140272994
}

\begin{abstract}
Instead of the per-survivor processing (PSP) detector which is the state-of-the-art solution for the joint data-channel estimation in fast fading channels, we propose a new reducedcomplexity maximum likelihood sequence detector (MLSD) based on the particle filtering technique. We show that the particle detector offers a better trade-off between performance and computational complexity, compared to a reduced-complexity PSP detector based on the decision-feedback sequence estimation (DFSE) algorithm. Moreover, the computational complexity of this new detector is adapted according to the signal-to-noise ratio.
\end{abstract}

\section{INTRODUCTION}

$\mathbf{I}$ $\mathrm{N}$ the mobile communication systems, the channel parameters are time-varying and unknown at the reception stage. In slow fading channels, the traditional approach to the detection problem is to provide a channel estimate for each data frame to a detector. But this method may not adequately track fast fading channels. The state-of-the-art solution for the detection problem in a fast fading channel is represented by the PerSurvivor Processing (PSP) detector, based on the adaptive Maximum Likelihood Sequence Estimation (MLSE) for the joint data-channel estimation. The channel estimation made by Least Mean Squares (LMS) [1], Recursive Least Squares (RLS) [2] or Kalman Filter (KF) [3] algorithms, is incorporated within the trellis of the Viterbi Decoder (VD), which decodes the information symbols. The main problem associated with this detector is the computational complexity of the VD. In fact, it might become quickly prohibitive in the communication systems which operate with high level modulations in long memory channels. We assume here that the dominant factor which determines the complexity of the VD is the channel memory.

Many researches have been conducted in order to reduce the computational complexity of the VD by selecting a subset of the states in the code trellis, as in the Reduced-State Sequence Estimation (RSSE) [4] and in the Decision-Feedback Sequence Estimation (DFSE) [5], or by selecting a subset of the paths in the code trellis, as in the M-algorithm [6] and in the T-algorithm [7].

In this paper, we keep the approach of the joint data-channel estimation used in the PSP detector and we propose a new suboptimal Maximum Likelihood (ML) detection algorithm, based

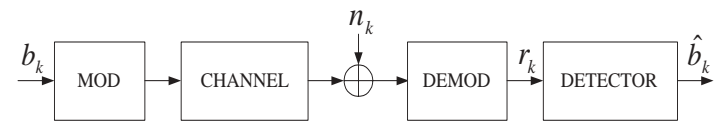

Fig. 1. Discrete-time equivalent lowpass transmission system model.

on the particle filtering technique [8]. The key idea is to explore only a subset of the possible transmitted sequences with a treesearch algorithm using particles. The selected sequences of the tree are the trajectories of particles, which evolve statistically in time according to the probability that a certain symbol was transmitted conditionally to the received signal. Along each trajectory, a KF estimates the Channel Impulse Response (CIR) coefficients.

This paper is organized as follows. In Section II the system model is presented. The structure of the particle detector is derived in Section III. Finally, simulation results are given in Section IV.

\section{SySTEM MODEL}

Fig. 1 shows the discrete-time equivalent lowpass transmission model considered in this paper. We will analyze only the binary modulation case. The generalization to more complex modulations is straightforward.

The information sequence is composed of independent and identically distributed bits. Each bit $b_{k}$ is transmitted within the symbol interval of duration $T$. The information bits are organized into frames composed of a preamble of known bits used in the estimation of the CIR, a block of information bits and a tail of known bits for properly terminating the trellis. The discrete-time channel model is represented by a symbolspaced Finite Impulse Response (FIR) filter, depicted on Fig. 2. The CIR coefficients $\left\{f_{k, l}\right\}_{l=0}^{L}$ where $L$ indicates the overall channel memory, are unknown at the reception stage. We assume that the time variations of the CIR coefficients into the data frame are important and therefore, the channel estimation provided using the preamble is insufficient. It represents only a initialization of the CIR coefficients estimates, which must be tracked even in the data frame. The matrix model of the 
received signal at the input of the detector is given by:

$$
\mathbf{r}_{k}=\mathbf{B} \cdot \mathbf{F}_{k}+\mathbf{n}_{k},
$$

where:

$$
\begin{aligned}
& \mathbf{r}_{k}=\left[\operatorname{Re}\left\{r_{k}\right\} \operatorname{Im}\left\{r_{k}\right\}\right], \\
& \mathbf{B}=\left[\begin{array}{lll}
b_{k} & b_{k-1} & \ldots \\
b_{k-L}
\end{array}\right]=\left[\begin{array}{ll}
b_{k} & \mathbf{B}_{k-1}
\end{array}\right], \\
& \mathbf{B}_{k-1}=\left[b_{k-1} \ldots b_{k-L}\right] \text {, } \\
& \mathbf{F}_{k}=\left[\begin{array}{cc}
\operatorname{Re}\left\{f_{k, 0}\right\} & \operatorname{Im}\left\{f_{k, 0}\right\} \\
\vdots & \vdots \\
\operatorname{Re}\left\{f_{k, L}\right\} & \operatorname{Im}\left\{f_{k, L}\right\}
\end{array}\right], \\
& \mathbf{n}_{k}=\left[\operatorname{Re}\left\{n_{k}\right\} \operatorname{Im}\left\{n_{k}\right\}\right] \text {. }
\end{aligned}
$$

The vector $\mathbf{n}_{k}$ is a discrete-time complex AWGN with zero mean, scalar variance $\sigma_{n}^{2}$ and independent real and imaginary components.

\section{THE PARTICLE DETECTOR}

The purpose of the detector examined in this paper is to estimate jointly the data sequence $B_{1}^{K}=\left\{b_{k}\right\}_{k=1}^{K}$, where $K$ is the number of information bits, and the CIR coefficients $\mathbf{F}=\left\{\mathbf{F}_{k}\right\}_{k=1}^{K}$. With this intention, we apply the ML principle and we estimate the data sequence as the sequence that maximizes the following probability:

$$
\hat{B}_{1}^{K}=\arg \max _{B_{1}^{K}} p\left(B_{1}^{K} \mid R_{1}^{K}, \hat{\mathbf{F}}\right),
$$

where $R_{1}^{K}=\left\{\mathbf{r}_{k}\right\}_{k=1}^{K}$ indicates the sequence of received samples, $\hat{\mathbf{F}}=\left\{\hat{\mathbf{F}}_{k \mid k-1}\right\}_{k=1}^{K}$ the sequence of the CIR coefficients estimates and $\hat{\mathbf{F}}_{k \mid k-1}$ the estimate of the CIR coefficients at time $k$ knowing the received samples until time $k-1$. For reasons of complexity, all the possible transmitted data sequences $B_{1}^{K}$ cannot be analyzed in order to calculate the a posteriori probability density in (2) and therefore, we consider the particle filtering approach to explore only a subset of these sequences. The particle filtering technique is a sequential Monte Carlo method used in non-linear/non-Gaussian tracking problems [8]. It is based upon point mass or particle representations of probability densities, which can be applied to any state

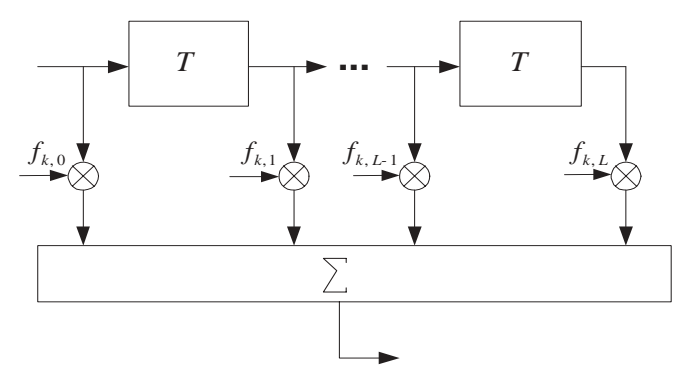

Fig. 2. Channel Model space model and which generalize the traditional Kalman filtering methods. In this case, the key idea is to approximate the a posteriori probability density in (2) with particles. This approximation is derived iteratively, getting to evolve the particles in time. The trajectory of a particle represents a possible transmitted data sequence. Moreover, we associate a Kalman Filter $(\mathrm{KF})$ to each particle trajectory in order to estimate the CIR coefficients jointly to the data. The particle filtering hypotheses for (1) are as follows:

- In order to derive the particle filtering equations, we keep the dependence of $b_{k}$ on the $L$ precedent bits, even if the data bits are independent and identically distributed:

$$
p\left(b_{k} \mid b_{1}, \cdots, b_{k-1}\right)=p\left(b_{k} \mid \mathbf{B}_{k-1}\right) ;
$$

- In a similar manner, $\mathbf{r}_{k}$ is independent given the transmitted bit at time $k$, the $L$ precedent transmitted bits and the estimate $\hat{\mathbf{F}}_{k \mid k-1}$ :

$$
p\left(\mathbf{r}_{k} \mid \mathbf{B}, \hat{\mathbf{F}}_{k \mid k-1}, A\right)=p\left(\mathbf{r}_{k} \mid \mathbf{B}, \hat{\mathbf{F}}_{k \mid k-1}\right) \quad \forall A .
$$

We can determinate the time evolution of the conditional probability $p\left(B_{1}^{K} \mid R_{1}^{K}, \hat{\mathbf{F}}\right)$ in two stages:

1) Prediction:

Calculation of the probability $p\left(B_{1}^{K} \mid R_{1}^{K-1}, \hat{\mathbf{F}}\right)$ from the probability $p\left(B_{1}^{K-1} \mid R_{1}^{K-1}, \hat{\mathbf{F}}\right)$;

\section{2) Correction:}

Calculation of the probability $p\left(B_{1}^{K} \mid R_{1}^{K}, \hat{\mathbf{F}}\right)$ from the probability $p\left(B_{1}^{K} \mid R_{1}^{K-1}, \hat{\mathbf{F}}\right)$.

For the first stage, applying the definition of conditional probability and considering that $B_{1}^{K}$ is a Markov process, we can write:

$$
\begin{gathered}
p\left(B_{1}^{K} \mid R_{1}^{K-1}, \hat{\mathbf{F}}\right) \\
=p\left(b_{K} \mid B_{1}^{K-1}, R_{1}^{K-1}, \hat{\mathbf{F}}\right) p\left(B_{1}^{K-1} \mid R_{1}^{K-1}, \hat{\mathbf{F}}\right) \\
=p\left(b_{K} \mid \mathbf{B}_{K-1}\right) p\left(B_{1}^{K-1} \mid R_{1}^{K-1}, \hat{\mathbf{F}}\right) .
\end{gathered}
$$

For the second stage, using the Bayes theorem and the hypothesis of independence of the received samples, the conditional probability $p\left(B_{1}^{K} \mid R_{1}^{K}, \hat{\mathbf{F}}\right)$ can be expressed as:

$$
\begin{gathered}
p\left(B_{1}^{K} \mid R_{1}^{K}, \hat{\mathbf{F}}\right) \\
=\frac{p\left(\mathbf{r}_{K} \mid B_{1}^{K}, R_{1}^{K-1}, \hat{\mathbf{F}}\right) p\left(B_{1}^{K} \mid R_{1}^{K-1}, \hat{\mathbf{F}}\right)}{p\left(\mathbf{r}_{K} \mid R_{1}^{K-1}, \hat{\mathbf{F}}\right)} \\
=\frac{p\left(\mathbf{r}_{K} \mid \mathbf{B}, \hat{\mathbf{F}}_{K \mid K-1}\right) p\left(B_{1}^{K} \mid R_{1}^{K-1}, \hat{\mathbf{F}}\right)}{\int p\left(\mathbf{r}_{K} \mid \mathbf{B}, \hat{\mathbf{F}}_{K \mid K-1}\right) p\left(B_{1}^{K} \mid R_{1}^{K-1}, \hat{\mathbf{F}}\right) d B_{1}^{K}} .
\end{gathered}
$$

By substitution of (3) into (4), (4) becomes:

$$
\begin{gathered}
p\left(B_{1}^{K} \mid R_{1}^{K}, \hat{\mathbf{F}}\right)= \\
\frac{p\left(\mathbf{r}_{K} \mid \mathbf{B}, \hat{\mathbf{F}}_{K \mid K-1}\right) p\left(b_{K} \mid \mathbf{B}_{K-1}\right) p\left(B_{1}^{K-1} \mid R_{1}^{K-1}, \hat{\mathbf{F}}\right)}{\int p\left(\mathbf{r}_{K} \mid \mathbf{B}, \hat{\mathbf{F}}_{K \mid K-1}\right) p\left(b_{K} \mid \mathbf{B}_{K-1}\right) p\left(B_{1}^{K-1} \mid R_{1}^{K-1}, \hat{\mathbf{F}}\right) d B_{1}^{K}}
\end{gathered}
$$


From (5), we can derive the classical result of the particle filtering technique. The conditional probability $p\left(B_{1}^{K} \mid R_{1}^{K}, \hat{\mathbf{F}}\right)$ is approximated by particles, characterized by a support $b_{k}$ evolving in time according to the transition probability $p\left(b_{K} \mid \mathbf{B}_{K-1}\right)$ and a weight depending on the probability $p\left(\mathbf{r}_{K} \mid \mathbf{B}, \hat{\mathbf{F}}_{K \mid K-1}\right)$ [8].

In this paper, we consider an original way to approximate the conditional probability $p\left(B_{1}^{K} \mid R_{1}^{K}, \hat{\mathbf{F}}\right)$ based on the particle filtering method named conditional drawing [9]. We observe that using the Bayes theorem, the property of Markov of the process $B_{1}^{K}$ and the hypothesis of independence of the received samples, we can write:

$$
\begin{gathered}
p\left(\mathbf{r}_{K} \mid \mathbf{B}, \hat{\mathbf{F}}_{K \mid K-1}\right) p\left(b_{K} \mid \mathbf{B}_{K-1}\right)= \\
p\left(\mathbf{r}_{K} \mid \mathbf{B}_{K-1}, \hat{\mathbf{F}}_{K \mid K-1}\right) p\left(b_{K} \mid \mathbf{B}_{K-1}, \hat{\mathbf{F}}_{K \mid K-1}, \mathbf{r}_{K}\right) .
\end{gathered}
$$

Therefore, (5) is equivalent to:

$$
\begin{gathered}
p\left(B_{1}^{K} \mid R_{1}^{K}, \hat{\mathbf{F}}\right)=\frac{p\left(\mathbf{r}_{K} \mid \mathbf{B}_{K-1}, \hat{\mathbf{F}}_{K \mid K-1}\right) \cdot}{\int p\left(\mathbf{r}_{K} \mid \mathbf{B}_{K-1}, \hat{\mathbf{F}}_{K \mid K-1}\right) \cdot} \\
\frac{\cdot p\left(b_{K} \mid \mathbf{B}_{K-1}, \hat{\mathbf{F}}_{K \mid K-1}, \mathbf{r}_{K}\right) p\left(B_{1}^{K-1} \mid R_{1}^{K-1}, \hat{\mathbf{F}}\right)}{\cdot p\left(b_{K} \mid \mathbf{B}_{K-1}, \hat{\mathbf{F}}_{K \mid K-1}, \mathbf{r}_{K}\right) p\left(B_{1}^{K-1} \mid R_{1}^{K-1}, \hat{\mathbf{F}}\right) d B_{1}^{K}} .
\end{gathered}
$$

Analogically to the classical result, we can approximate the conditional probability $p\left(B_{1}^{K} \mid R_{1}^{K}, \hat{\mathbf{F}}\right)$ with particles, which evolve in time according to the conditional transition probability $p\left(b_{K} \mid \mathbf{B}_{K-1}, \hat{\mathbf{F}}_{K \mid K-1}, \mathbf{r}_{K}\right)$ and have a weight depending on the probability $p\left(\mathbf{r}_{K} \mid \mathbf{B}_{K-1}, \hat{\mathbf{F}}_{K \mid K-1}\right)$. First, we will calculate the conditional transition probability $p\left(b_{K} \mid \mathbf{B}_{K-1}, \hat{\mathbf{F}}_{K \mid K-1}, \mathbf{r}_{K}\right)$ and then the weight of a particle.

In the binary modulation case, the support of the particles can assume the values $b_{K}=1$ or $b_{K}=-1$ and hence, we must determine the two conditional transition probabilities $p\left(b_{K}=\right.$ $\left.1 \mid \mathbf{B}_{K-1}, \hat{\mathbf{F}}_{K \mid K-1}, \mathbf{r}_{K}\right)$ and $p\left(b_{K}=-1 \mid \mathbf{B}_{K-1}, \hat{\mathbf{F}}_{K \mid K-1}, \mathbf{r}_{K}\right)$. For simplicity, we consider only the probability for the bit $b_{K}=1$. Applying the Bayes theorem, the conditional transition probability is given by:

$$
\begin{gathered}
p\left(b_{K}=1 \mid \mathbf{B}_{K-1}, \hat{\mathbf{F}}_{K \mid K-1}, \mathbf{r}_{K}\right) \\
=\frac{p\left(\mathbf{r}_{K} \mid \mathbf{B}_{+}, \hat{\mathbf{F}}_{K \mid K-1}\right) p\left(b_{K}=1 \mid \mathbf{B}_{K-1}, \hat{\mathbf{F}}_{K \mid K-1}\right)}{\int p\left(\mathbf{r}_{K} \mid \mathbf{B}, \hat{\mathbf{F}}_{K \mid K-1}\right) p\left(b_{K} \mid \mathbf{B}_{K-1}, \hat{\mathbf{F}}_{K \mid K-1}\right) d b_{K}},
\end{gathered}
$$

where we have defined $\mathbf{B}_{+}=\left[\begin{array}{ll}b_{K}=1 & \mathbf{B}_{K-1}\end{array}\right]$ and similarly $\mathbf{B}_{-}=\left[b_{K}=-1 \quad \mathbf{B}_{K-1}\right]$. Owing to the independence and the equally probability of the information bits, we can write:

$$
\begin{gathered}
p\left(b_{K}=1 \mid \mathbf{B}_{K-1}, \hat{\mathbf{F}}_{K \mid K-1}\right) \\
=p\left(b_{K}=-1 \mid \mathbf{B}_{K-1}, \hat{\mathbf{F}}_{K \mid K-1}\right)=\frac{1}{2} .
\end{gathered}
$$

Therefore, (8) becomes:

$$
p\left(b_{K}=1 \mid \mathbf{B}_{K-1}, \hat{\mathbf{F}}_{K \mid K-1}, \mathbf{r}_{K}\right)
$$

$$
=\frac{p\left(\mathbf{r}_{K} \mid \mathbf{B}_{+}, \hat{\mathbf{F}}_{K \mid K-1}\right)}{p\left(\mathbf{r}_{K} \mid \mathbf{B}_{+}, \hat{\mathbf{F}}_{K \mid K-1}\right)+p\left(\mathbf{r}_{K} \mid \mathbf{B}_{-}, \hat{\mathbf{F}}_{K \mid K-1}\right)} .
$$

We observe that the probability densities $p\left(\mathbf{r}_{K} \mid \mathbf{B}_{+}, \hat{\mathbf{F}}_{K \mid K-1}\right)$ and $p\left(\mathbf{r}_{K} \mid \mathbf{B}_{-}, \hat{\mathbf{F}}_{K \mid K-1}\right)$ depend on the estimate $\hat{\mathbf{F}}_{K \mid K-1}$ of the CIR coefficients and hence, on the evolution model of the $\mathrm{KF}$ used for the channel estimation. We have chosen a simple model to represent the channel evolution. More complex models could improve the channel estimation. It is expressed by the following equation:

$$
\mathbf{F}_{k}=\mathbf{F}_{k-1}+\mathbf{W}_{k} \quad \text { for } k=1, \cdots, K,
$$

where the matrix $\mathbf{W}_{k}$, defined as:

$$
\mathbf{W}_{k}=\left[\begin{array}{cc}
\operatorname{Re}\left\{w_{k, 0}\right\} & \operatorname{Im}\left\{w_{k, 0}\right\} \\
\vdots & \vdots \\
\operatorname{Re}\left\{w_{k, L}\right\} & \operatorname{Im}\left\{w_{k, L}\right\}
\end{array}\right]
$$

is a complex discrete-time AWGN with zero-mean and covariance represented by the $(L+1) \times(L+1)$ matrix $\mathbf{Q}$. Considering the channel evolution model (11) and the observation model (1), the KF equations for the channel estimation at time $k$ are divided in a prediction phase:

$$
\begin{aligned}
\hat{\mathbf{F}}_{k \mid k-1} & =\hat{\mathbf{F}}_{k-1 \mid k-1} \\
\tilde{\mathbf{P}}_{k \mid k-1} & =\tilde{\mathbf{P}}_{k-1 \mid k-1}+\mathbf{Q}
\end{aligned}
$$

and a correction phase:

$$
\begin{aligned}
\hat{\mathbf{F}}_{k \mid k} & =\hat{\mathbf{F}}_{k \mid k-1}+\mathbf{G}_{k}\left(\mathbf{r}_{k}-\mathbf{B} \hat{\mathbf{F}}_{k \mid k-1}\right) \\
\tilde{\mathbf{P}}_{k \mid k} & =\tilde{\mathbf{P}}_{k \mid k-1}-\mathbf{G}_{k} \mathbf{B} \tilde{\mathbf{P}}_{k \mid k-1},
\end{aligned}
$$

where the estimate $\hat{\mathbf{F}}_{k \mid k}$ is given by:

$$
\hat{\mathbf{F}}_{k \mid k}=E \mathbf{F}_{k} \mid R_{1}^{k}
$$

the matrix $\tilde{\mathbf{P}}_{k \mid k}$ is the covariance of the error of the KF, defined as:

$$
\tilde{\mathbf{F}}_{k \mid k}=\mathbf{F}_{k}-\hat{\mathbf{F}}_{k \mid k}
$$

the quantity:

$$
\mathbf{G}_{k}=\tilde{\mathbf{P}}_{k \mid k-1} \mathbf{B}^{T}\left(\mathbf{B} \tilde{\mathbf{P}}_{k \mid k-1} \mathbf{B}^{T}+\sigma_{n}^{2}\right)^{-1}
$$

is the Kalman gain and $H^{T}$ is the transpose matrix of $H$. By substitution of (16) into (1), (1) becomes:

$$
\mathbf{r}_{K}=\mathbf{B} \tilde{\mathbf{F}}_{K \mid K-1}+\mathbf{B} \hat{\mathbf{F}}_{K \mid K-1}+\mathbf{n}_{K}
$$

Thanks to (18) and since the noises $\mathbf{n}_{K}$ and $\mathbf{W}_{K}$ are gaussian with zero-mean, we notice that the probability density $p\left(\mathbf{r}_{K} \mid \mathbf{B}, \hat{\mathbf{F}}_{K \mid K-1}\right)$ is gaussian with mean:

$$
E\left\{\mathbf{r}_{K} \mid \mathbf{B}, \hat{\mathbf{F}}_{K \mid K-1}\right\}=\mathbf{B} \hat{\mathbf{F}}_{K \mid K-1}
$$


and scalar variance:

$$
\begin{gathered}
E\left\{\left(\mathbf{r}_{K}-\mathbf{B} \hat{\mathbf{F}}_{K \mid K-1}\right)\left(\mathbf{r}_{K}-\mathbf{B} \hat{\mathbf{F}}_{K \mid K-1}\right)^{T} \mid \mathbf{B}, \hat{\mathbf{F}}_{K \mid K-1}\right\} \\
=E\left\{\left(\mathbf{B} \tilde{\mathbf{F}}_{K \mid K-1}+\mathbf{n}_{K}\right)\left(\mathbf{B} \tilde{\mathbf{F}}_{K \mid K-1}+\mathbf{n}_{K}\right)^{T} \mid \mathbf{B}, \hat{\mathbf{F}}_{K \mid K-1}\right\} \\
=\mathbf{B} \tilde{\mathbf{P}}_{K \mid K-1} \mathbf{B}^{T}+\sigma_{n}^{2} .
\end{gathered}
$$

Hence, the expression of the conditional probability density of the observation $\mathbf{r}_{K}$ is expressed by:

$$
\begin{gathered}
p\left(\mathbf{r}_{K} \mid \mathbf{B}, \hat{\mathbf{F}}_{K \mid K-1}\right)=\frac{1}{2 \pi \sqrt{\mathbf{B} \tilde{\mathbf{P}}_{K \mid K-1} \mathbf{B}^{T}+\sigma_{n}^{2}}} \exp \left\{-\frac{1}{2} .\right. \\
\left.\left(\mathbf{r}_{K}-\mathbf{B} \hat{\mathbf{F}}_{K \mid K-1}\right)\left(\mathbf{B} \tilde{\mathbf{P}}_{K \mid K-1} \mathbf{B}^{T}+\sigma_{n}^{2}\right)^{-1}\left(\mathbf{r}_{K}-\mathbf{B} \hat{\mathbf{F}}_{K \mid K-1}\right)^{T}\right\} .
\end{gathered}
$$

In order to calculate the weight of a particle, we rewrite (7) from time $K-1$ to 1 :

$$
\begin{gathered}
p\left(B_{1}^{K} \mid R_{1}^{K}, \hat{\mathbf{F}}\right)=\frac{p\left(\mathbf{r}_{K} \mid \mathbf{B}_{K-1}, \hat{\mathbf{F}}_{K \mid K-1}\right) \cdots p\left(\mathbf{r}_{1} \mid \mathbf{B}_{0}, \hat{\mathbf{F}}_{1 \mid 0}\right) .}{\int p\left(\mathbf{r}_{K} \mid \mathbf{B}_{K-1}, \hat{\mathbf{F}}_{K \mid K-1}\right) \cdots p\left(\mathbf{r}_{1} \mid \mathbf{B}_{0}, \hat{\mathbf{F}}_{1 \mid 0}\right)} \\
\frac{\cdot p\left(b_{K} \mid \mathbf{B}_{K-1}, \hat{\mathbf{F}}_{K \mid K-1}, \mathbf{r}_{K}\right) \cdots p\left(b_{1} \mid \mathbf{B}_{0}, \hat{\mathbf{F}}_{1 \mid 0}, \mathbf{r}_{1}\right)}{\cdot p\left(b_{K} \mid \mathbf{B}_{K-1}, \hat{\mathbf{F}}_{K \mid K-1}, \mathbf{r}_{K}\right) \cdots p\left(b_{1} \mid \mathbf{B}_{0}, \hat{\mathbf{F}}_{1 \mid 0}, \mathbf{r}_{1}\right) d B_{1}^{K}} .
\end{gathered}
$$

We use the particle approximation for the terms related to the particle evolution:

$$
\begin{gathered}
p\left(b_{K} \mid \mathbf{B}_{K-1}, \hat{\mathbf{F}}_{K \mid K-1}, \mathbf{r}_{K}\right) \ldots p\left(b_{1} \mid \mathbf{B}_{0}, \hat{\mathbf{F}}_{1 \mid 0}, \mathbf{r}_{1}\right) \simeq \\
\sum_{n=1}^{N_{p}} \frac{1}{N_{p}} \delta\left(b_{K}-b_{K}^{n}\right) \ldots \delta\left(b_{1}-b_{1}^{n}\right),
\end{gathered}
$$

where $N_{p}$ is the number of particles and $b_{k}^{i}$ is the support of the particle $i$ at time $k$. We notice that the terms related to the particle evolution are taken into account in the conditional transition probability calculation and consequently, don't influence the weight determination. By substitution of (23) into (22), (22) becomes:

$$
\begin{gathered}
p\left(B_{1}^{K} \mid R_{1}^{K}, \hat{\mathbf{F}}\right) \simeq \\
\sum_{n=1}^{N_{p}} \frac{p\left(\mathbf{r}_{K} \mid \mathbf{B}_{K-1}^{n}, \hat{\mathbf{F}}_{K \mid K-1}^{n}\right) \cdots p\left(\mathbf{r}_{1} \mid \mathbf{B}_{0}^{n}, \hat{\mathbf{F}}_{1 \mid 0}^{n}\right)}{\sum_{m=1}^{N_{p}} p\left(\mathbf{r}_{K} \mid \mathbf{B}_{K-1}^{m}, \hat{\mathbf{F}}_{K \mid K-1}^{m}\right) \cdots p\left(\mathbf{r}_{1} \mid \mathbf{B}_{0}^{m}, \hat{\mathbf{F}}_{1 \mid 0}^{m}\right)} . \\
\cdot \delta\left(b_{K}-b_{K}^{n}\right) \cdots \delta\left(b_{1}-b_{1}^{n}\right),
\end{gathered}
$$

where the term multiplying the Dirac delta represents the weight of the particle $n$ at time $K$. This weight can be calculated recursively with the expression:

$$
\rho_{k}^{n}=\frac{\rho_{k-1}^{n} p\left(\mathbf{r}_{k} \mid \mathbf{B}_{k-1}^{n}, \hat{\mathbf{F}}_{k \mid k-1}^{n}\right)}{\sum_{m=1}^{N_{p}} \rho_{k-1}^{m} p\left(\mathbf{r}_{k} \mid \mathbf{B}_{k-1}^{m}, \hat{\mathbf{F}}_{k \mid k-1}^{m}\right)},
$$

for $k=1, \ldots, K$ with the initial condition $\rho_{0}^{n}=1 / N_{p}$ for $n=1, \ldots, N_{p}$. In (25), the probability $p\left(\mathbf{r}_{k} \mid \mathbf{B}_{k-1}^{n}, \hat{\mathbf{F}}_{k \mid k-1}^{n}\right)$ can be determined in the following way:

$$
p\left(\mathbf{r}_{k} \mid \mathbf{B}_{k-1}^{n},, \hat{\mathbf{F}}_{k \mid k-1}^{n}\right)
$$

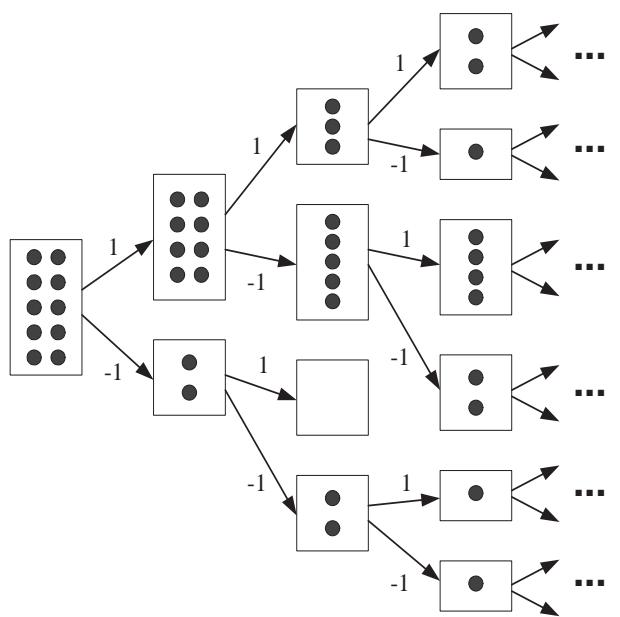

Fig. 3. Tree of the particle MLSD.

$$
\begin{aligned}
& =\int p\left(\mathbf{r}_{k} \mid b_{k}^{n}, \mathbf{B}_{k-1}^{n}, \hat{\mathbf{F}}_{k \mid k-1}^{n}\right) p\left(b_{k}^{n} \mid \mathbf{B}_{k-1}^{n}, \hat{\mathbf{F}}_{k \mid k-1}^{n}\right) d b_{k}^{n} \\
& =\frac{1}{2} p\left(\mathbf{r}_{k} \mid \mathbf{B}_{+}^{n}, \hat{\mathbf{F}}_{k \mid k-1}^{n}\right)+\frac{1}{2} p\left(\mathbf{r}_{k} \mid \mathbf{B}_{-}^{n}, \hat{\mathbf{F}}_{k \mid k-1}^{n}\right) .
\end{aligned}
$$

In order to develop a particle MLSD based on the conditional drawing technique, we use a tree-search algorithm to explore the space of the possible transmitted sequences. The root of the tree consists in a group holding all the $N_{p}$ particles. Initially, the particles are equally weighted. The first bit to be estimated can assume the values 1 or -1 and hence, the particles divide in two groups proportionally to the conditional transition probabilities $p\left(b_{1}=1 \mid \mathbf{B}_{0}, \mathbf{r}_{1}, \tilde{\mathbf{F}}_{1 \mid 0}\right)$ and $p\left(b_{1}=-1 \mid \mathbf{B}_{0}, \mathbf{r}_{1}, \tilde{\mathbf{F}}_{1 \mid 0}\right)$, where $\mathbf{B}_{0}$ corresponds to the last $L$ bits of the preamble sequence and the estimate $\tilde{\mathbf{F}}_{1 \mid 0}$ is calculated applying the KF on the preamble sequence. At the next instant the particles will be divided in four groups. So the groups of particles form the nodes of a tree. An example of a particle tree is represented in Fig. 3. We analyze in details the generic transition process from time $k-1$ to time $k$. For each group at time $k-1$, we calculate the conditional transition probabilities $p\left(b_{k}=1 \mid \mathbf{B}_{k-1}, \mathbf{r}_{k}, \tilde{\mathbf{F}}_{k \mid k-1}\right)$ and $p\left(b_{k}=-1 \mid \mathbf{B}_{k-1}, \mathbf{r}_{k}, \tilde{\mathbf{F}}_{k \mid k-1}\right)$, given by (10). After the division of the particles for each group at time $k-1$ proportionally to the conditional transition probability, the empty groups are eliminated. For the survivor groups at time $k$, we calculate the estimate $\tilde{\mathbf{F}}_{k \mid k-1}$ according to (13) and (14) and the weight associated with a particle according to (25). We notice that the particles in a group have the same weight and that the weight of a group is equal to the product between the number of particles in the group and the weight of a particle.

As in the VD, the decision on the information bits in the particle MLSD is carried out after the processing of $5 L$ received samples. The particle detector estimates at time $k+5 L$ the bit at time $k$. This corresponds to the bit $b_{k}$ of the maximum weight group at time $k+5 L$. At the end of the information sequence, the particle algorithm must be terminated with the $L$ 
known bits of the tail sequence in order to finish in a known final state. In this closing phase, the division of the particles in groups is deterministic; the particles transfer in the group corresponding to the known transition bit. Moreover, the calculation of the weights is modified. In (26), only the probability associated to the known bit is considered, because the other is equal to zero. In the particle detector described above, the particles are initially concentrated in one group and during the processing of each received sample, they are spread in the space of the possible transmitted sequences. The maximum degree of exploration of this space is given by the number of particles. When each group contains only one particle, some particles can explore improbable zones. In order to improve the exploration around the most probable zones, we can force a particle redistribution. The redistribution is a very critical task, because the performance strongly depends on it. For example, we can redistribute the particles every $L$ bits: the particles in groups with a weight inferior to a given threshold are moved in the group with maximum weight.

\section{Simulation RESUlts}

In this section, we present simulation results depicting the performance of the proposed particle detector. The adopted performance measure is the Frame Error Rate (FER) versus the Signal-to-Noise Ratio (SNR) $E_{b} / N_{0}$, where $E_{b}$ denotes the average bit energy and $N_{0}$ the unilateral power spectral density of the noise. We determine the performance for a Global System for Mobile communications (GSM) system. We assume that the receiver detects only a slot for each Time Division Multiple Access (TDMA) frame, constituted by 8 slots. A GSM slot consists in two burst of 58 information bits separated by a midamble sequence of 26 known bits. Reference [10] shows that the backward detection of the first burst and the forward detection of the second burst give approximately the same performance. Therefore, we can consider only the forward detection of the second burst. The modulation scheme corresponds to a discrete-time linearized representation of a Gaussian Minimum Shift Keying (GMSK) signal [11], in order to simplify the structure of the demodulator. By means of a shift phase, the received signal at the input of the detector is described by (1). For the generation of the channel coefficients, unknown by the receiver, we consider two models. In the first model, the channel memory $L$ is equal to 7 and the channel coefficients are given by:

$$
\begin{aligned}
\operatorname{Re}\left\{f_{k, l}\right\} & =a_{l} \cos \left(2 \pi f_{d, l} k T_{s}\right) \\
\operatorname{Im}\left\{f_{k, l}\right\} & =a_{l} \sin \left(2 \pi f_{d, l} k T_{s}\right),
\end{aligned}
$$

for $l=0, \ldots, 7$. The amplitudes are chosen in order to obtain a phase-minimal channel with unitary energy:

$$
\left[a_{0}, \ldots, a_{7}\right]=[0.56,0.49,0.42,0.35,0.28,0.21,0.14,0.07] \text {. }
$$

The Doppler frequencies in $\mathrm{Hz}$ associated with each channel path are:

$$
\left[f_{d, 0}, \ldots, f_{d, 7}\right]=[10,20,30,40,50,60,70,80] .
$$

The sampling period $T_{s}$ is equal to the symbol interval $T=$ $3.69 \mu \mathrm{s}$. For the second model, we consider the 12-tap Hilly Terrain (HT) GSM channel model, described in [12]. The coefficients $\left\{f_{k, l}\right\}_{l=0}^{L}$ are generated using a bank of time-shifted independent Rayleigh flat fading channel simulators. In each simulator, a white complex Gaussian noise passes through a digital second-order low-pass Chebyshev filter followed by a fifthorder Butterworth filter, which impart the Rayleigh Doppler spectrum. In simulations, we consider fast fading conditions and hence, we assume a Doppler frequency for each path equal to $200 \mathrm{~Hz}$. This corresponds to a vehicle speed of $240 \mathrm{~km} / \mathrm{h}$ for a $900 \mathrm{MHz}$ GSM system. The channel memory $L$ of a HT channel is equal to 6 .

We compare the performance of the particle detector with the performance of the PSP detector. For the complexity reduction of this receiver, we use the DFSE algorithm, which presents a reduced number of states in the Viterbi trellis. The overall channel memory is considered and the terms of residual ISI are corrected along each survivor path. Fig. 4 shows the performance obtained for the first channel model. If we reduce the number of the Viterbi states of the PSP detector, we observe a frame error floor at $4 \cdot 10^{-2}$. This behavior is absent in the particle detector; the performance of the particle detector with 128 particles and with 8 particles are very close. The computational complexity of these different detectors for the first channel model is depicted in Fig. 5. Unlike the PSP detector, the computational complexity of the PD is adapted according to the quality of the received signal. As a consequence, for the same performance, except at low $E_{b} / N_{0}$, the complexity of the PD is always lower than the PSP detector. The performance and the computational complexity for the HT240 channel model are respectively given in Fig. 6 and in Fig. 7. Like previously, we observe a frame error floor at $10^{-1}$ if we reduce the number of states of PSP detector to 8 states. At high $E_{b} / N_{0}$, the performance of the PD with 8 and 64 particles are noticeably worse than that of a PSP detector with 16 states. However, the computational complexity of the PD is more than 10 times lower than the PSP detector.

\section{CONCLUSION}

A reduced-complexity MLSD based on the particle filtering technique has been proposed and analyzed. The particles arranged in groups statistically explore the space of the possible transmitted sequences forming a tree. The number of paths examined by groups of particles depends on the quality of the received signal. In fact, for high SNR the particles remain concentrated in one group only, whereas for low SNR they divide into several groups. This means that the PD complexity is lower than that of a detector implemented using a Viterbi algorithm for a equal number of particles and states. Moreover, if we reduce strongly the computational complexity, the PD has shown better performance than that of a PSP detector for the same number of particles and states. Hence, we can conclude that the PD represents a very good trade-off between error rate 


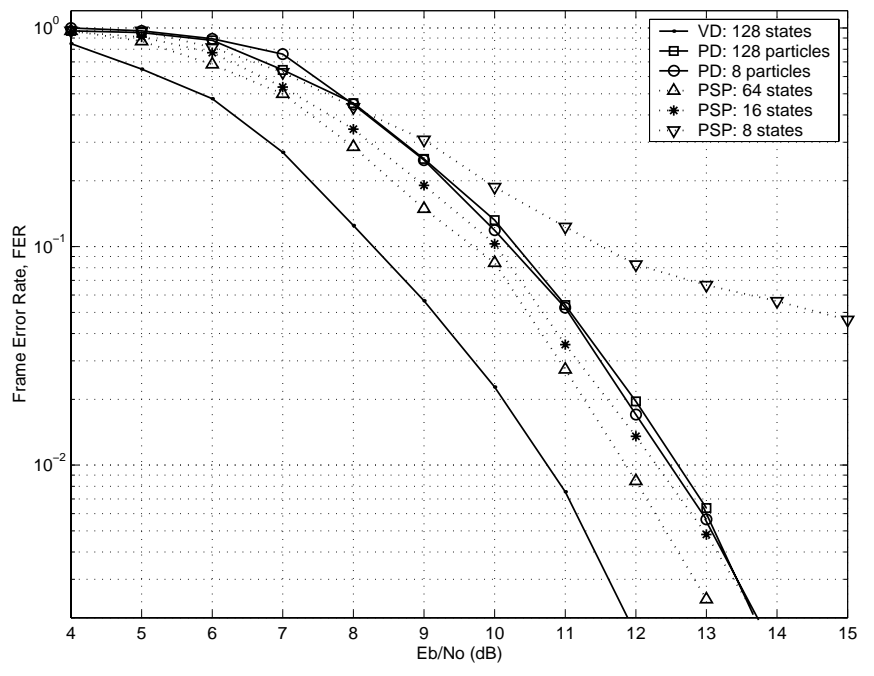

Fig. 4. FER versus $E_{b} / N_{0}$ for the first channel model.

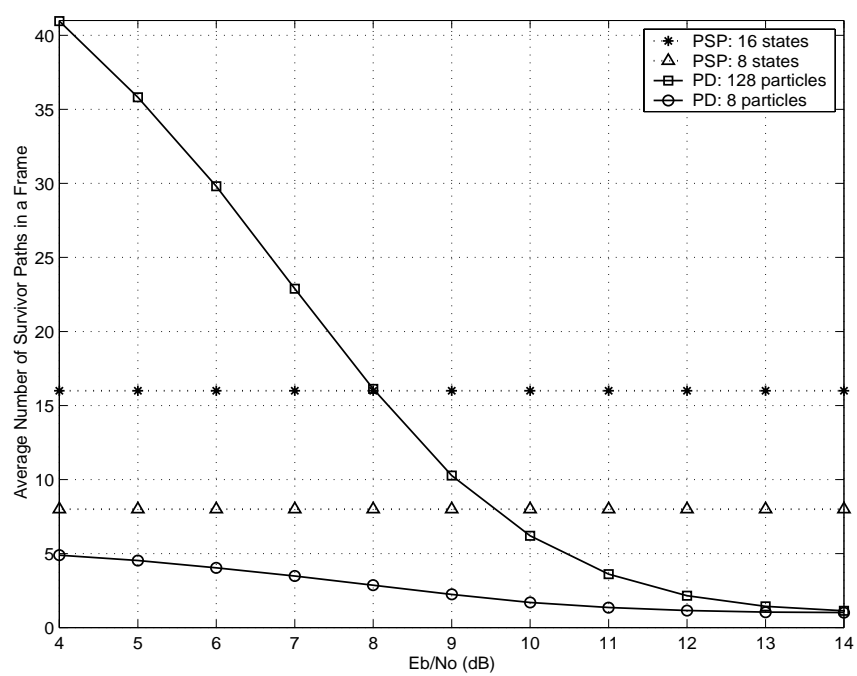

Fig. 5. Computational complexity for the first channel model.

performance and computational complexity especially for communication systems operating with high level modulations and over long memory channels.

\section{REFERENCES}

[1] C. -K. Tzou, R. Raheli and A. Polydoros, "Applications of Per-Survivor Processing to mobile digital communications," Proc. IEEE Globecom, Nov. 1993.

[2] P. Castoldi, R. Raheli and G. Marino, "Efficient trellis search algorithms for adaptive MLSE on fast Rayleigh fading channels," Proc. IEEE Globecom, Nov. 1994.

[3] M. J. Omidi, S. Pasupathy and P. G. Gulak, "Joint data and Kalman estimation for Rayleigh fading channels," Journal of Wireless Pers. Com., Kluwers Publishers, 1998.

[4] M. V. Eyuboğlu and S. U. H. Qureshi, "Reduced-state sequence estimation with set partitioning and decision feedback," IEEE Trans. on Com., Vol. 36, pp. 13-20, Jan. 1988.

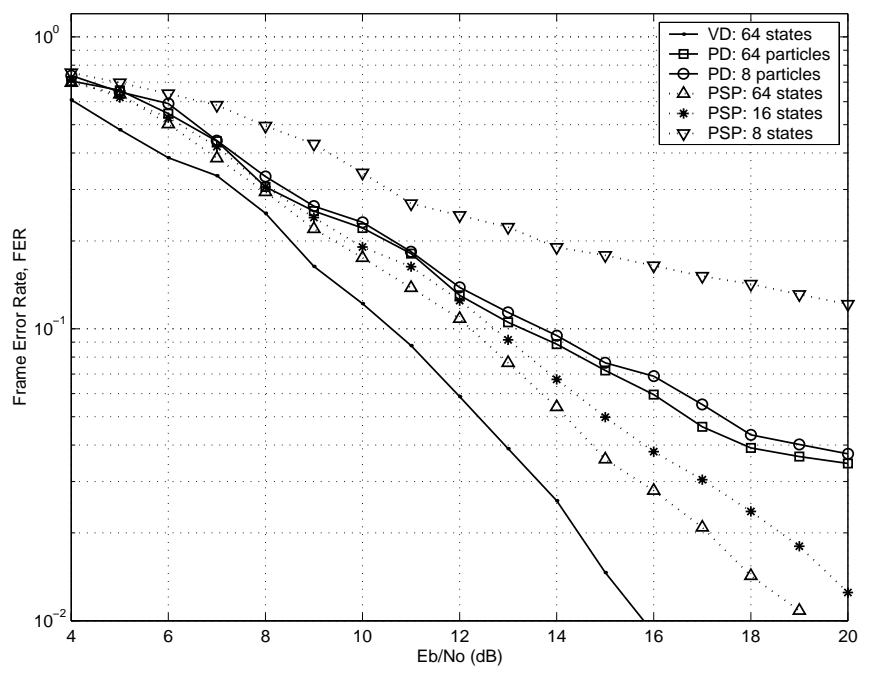

Fig. 6. FER versus $E_{b} / N_{0}$ for the HT240 channel.

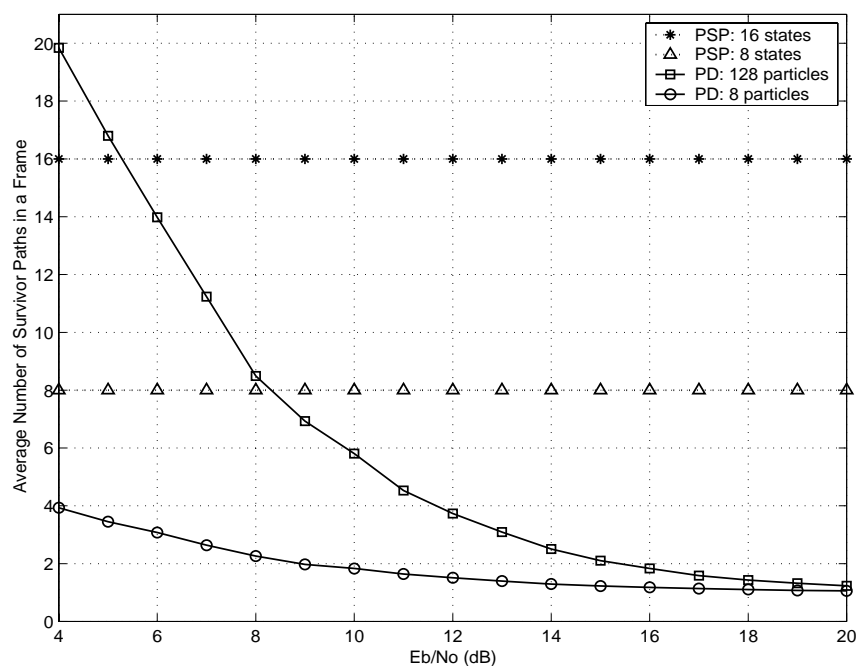

Fig. 7. Computational complexity for the HT240 channel.

[5] A. Duel-Hallen and C. Heegard, "Delayed decision-feedback sequence estimation," IEEE Trans. on Com., Vol. 37, pp. 428-436, May 1989.

[6] J. B. Anderson and S. Mohan, "Sequential coding algorithm: a survey and cost analysis," IEEE Trans. on Com., Vol. 32, pp. 169-176, Feb. 1984.

[7] S. J. Simmons, "Breadth-first trellis decoding with adaptive effort," IEEE Trans. on Com., Vol. 38, pp. 3-12, Jan. 1990.

[8] A. Doucet, J. F. G. de Freitas and N. J. Gordon, Sequential Monte Carlo methods in practice. New York: Springer-Verlag, 2001.

[9] G. Rigal, Non linear filtering, particle resolution and signal processing applications (in french). Ph.D. thesis, Laboratory for Analysis and Architecture of Systems (LAAS), France, 1993.

[10] J. Salo and N. Nefedov, "Performance evaluation of a per-survivor processing soft-output detector in GSM EDGE environment," Proc. of Finnish Wireless Com. Workshop (FWCW 2000), pp. 7-11, Oulu, 29-30 May 2000.

[11] P. A. Laurent, "Exact and approximate construction of digital phase modulation by superposition of amplitude modulated pulses (AMP)," IEEE Trans. on Com., Vol. 34, pp. 150-160, Feb. 1986.

[12] GSM Recommandations 05.05 Version 6.3.0 Release 1997, "Digital cellular telecommunications system (Phase $2+$ ); radio transmission and reception." 